\title{
The technical research of achieving the internet of things based on cloud computing platform
}

\author{
HaiYan Huang \\ Institute of information science and technology Jiujiang University \\ ,JiuJiang,China \\ goodhelen406@163.com
}

Keywords: internet of things, cloud computing, framework design

\begin{abstract}
Analyzed the relationship between cloud computing and the internet of things, Cloud computing has unique advantages, so it becomes the main supporting technologies of large-scale, cross regional development,and study on network framework based on cloud computing, and proposed the key technology to realize this framework design.
\end{abstract}

\section{Introduction}

With the development of computer technology and mobile communications technology matures,the concept attracted quickly the attention of countries around the world after the Massachusetts Institute of Technology presented the concept of the Internet of Things in 1999,Many countries have begun to develop appropriate networking strategy.For this reason,The International Telecommunication Union has set up a working group of international experts pan-social network on WSIS(World Summit on the Information Society)held in Geneva in the April 8, 2005,the working group is to provide a consultancy to discuss about the Internet of things.For example:The EU has developed 《Internet of things" of plan of the EU action,and have made clear development roadmap of Internet of things of the three stage of 2010,2015,2020; The Japanese government proposed a U-Japan, $\mathrm{U}$ is ubiquitous,meaning of ubiquitous is omnipresent,it means that all resources in Japan will soon be connected through the network communication technology, sensor technology, etc[1]; The information and communication of korean faced new global industrial revolution tomake some "U-Korea" strategy,based on wireless sensor networks , connectting all available resources Korea to share and use for people and promote economic development in Korea;In China, internet of things has been included in the "Twelfth Five-Year Plan", is one of China's strategic emerging industries,to meet the demand for talent rapid development of the Internet of Things, our colleges and universities have also been set up networking professional.As a new computing models and services of internet development,Cloud Computing also attracted the attention of the world's countries.All the countries in the world announced relevant policies to support cloud computing industry.Cloud computing has a very large scale, virtualization, high reliability, versatility, scalability, cost, etc,it is one of the necessary technolog of cross-sectoral, cross-regional development of internet of things,so,we need to build cloud platform to operator the internet of things.

\section{The concept of cloud computing}

Eric Emerson Schmidt who is google CEO first proposed the concept of "cloud computing" in search engine assembly in august 9, 2006,it immediately attracted wide attention.So far,cloud computing is already a mature technology widely used.People unwittingly use some convenient self-service provided by cloud computingIn real life.For example:google, amazon,microsoft office 365 etc. are typical applications of cloud computing.People simply enter keywords in google engine and information that is distributed storage on the computer on around the world is matched based on a unique method of calculation,and find the corresponding information.And people do not need to concern about what is the use of hardware, software in the end.This is the charm of cloud computing.Cloud computing defined by the U.S. National Institute of Standards and 
Technology:Cloud computing is a pay-per-use model,this mode provides available, convenient, on-demand network access,these resources can be quickly provide into the shared pool of configurable computing resources.,putting in seldom management effort or little interaction with service providers, These resources can be quickly provide[2].Emergence of cloud computing has changed the traditional way of working of many enterprises.In the past,most businesses need to buy a lot of hardware and software that are infrastructure to handle these data,then spend a lot of mone to send someone to maintain the hardware and software.Now these companies simply need to spend a small amount of rent, when to hire these software services,no need to purchase hardware and software you can enjoy the service,this method can greatly save the resources.people can used computer resources as cheap as water, electricity, gas.This new IT Revolution freed people from the tedious maintenance work, Thus there are a lot of time to think about the innovation of the project.

The featuresof cloud computing. According to the concept and current applications of cloud computing,the main features of cloud computing can be summarized as follows:

The firs:Sharing a common resource pool.Cloud computing integrates amounts of computer resources to manage and maintain.Users can access the required resources through the terminal without having to purchase relevant computer hardware and software[3];

The secend:Users demand access, by using the appropriate amount of rent paid.Because users do not have to purchase computer hardware and software for data processing,just pay a small amount of rent, you can gain access to computer resources required,this approach greatly reduces the user's cost,and users can also extend the growing demand for services,so this improves the utilization of funds;

The third:Users can easily access cloud computing services.Users can access cloud computing services through personal computers, smart phones and other devices ;

The fourth:High reliability.Cloud computing services can avoid data corruption by viruses.Because cloud computing storage equipment is very reliable to help users to manage data,and there are so many Excellent staff to help you maintain data,so users do not worry about your data being lost or destroyed[4].

It is because of the characteristics of cloud computing of the above,individuals can easily enjoy cloud computing services.For example:Personal photos, friends contact etc are stored into the "cloud" and backup data,data can also be found in case of a system crash.Companies can also enjoy spending a low-cost cloud computing services.In addition to,companies can also own private cloud,that is the integration of internal all resources, centralized management by cloud computing service for enterprises and other companies to share their own.this improves the utilization of corporate IT resources.cloud computing makes both individuals and enterprises to handle the needs of life, work,it is very Convenient and efficient,So the cloud computing has been rapidly applied in short time.

\section{The concept of internet of things}

The concept of things early is proposed in mobile computing and network of international conferences held in 1999 by the United States.At that time in China,it is called sensor network.In 2005, the World Summit on the Information Society held in Tunis, the International Telecommunication Union formally proposed the concept of the Internet of Things.The concept of things from the beginning attracted global attention. The development of the Internet of things has been referred to as one of the strategic new industries.of the economic development of countries.In China, the development of things is included in the "Twelfth Five-Year" development plan.So what are the internet of things?Various experts on the concept of things have a different understanding and definitions,collective on the Internet of things define summarized as follows:The Internet of things is the extension and expansion of the Internet network,Based on Radio frequency identification (RFID) technology, global positioning systems, infrared sensors, and other that are technology-based devices to get location,sound,light, and other information of items connected to the Internet.these information is processed through a variety of transmission network to the specified information processing center,in order to achieve the real-time monitoring, locating, managing, controlling of these items at any time and any place[5].The conceptual model of internet of things as shown in Figure one: 


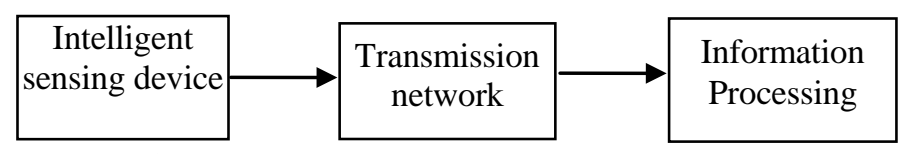

Fig.1The conceptual model of internet of things

Intelligent sensing devices that like the eyes, ears, nose and limbs and other access the object information.Transmission transmission network like the nervous system and transfer the collected information to the information processing center.And the information processing center is like the human brain.

In china,in the article of 《Internet of things of Twelfth Five Year "development planning》pointed out: : At present, China's Internet of things have been used in the field of security,power, transportation, logistics, medical etc.Among them, the field of security applications have been taken to the good results, other areas of application are evolving.

Cloud computing and the Internet of things. The Internet of things is applied in various industries through the sensing technology, communication technology, data fusion and intelligent technology and nanotechnology. The collected information is respectively connected objects through the sensing technology.For example:reading the basic information of object by scanning electronic label through radio frequency identification technology,connecting each object to the internet through wired, wireless and other network communications technologies for all users to share.carring on corresponding processing data through data fusion and intelligent technology, in order to meet the user's requirements,and one of the most important technique cloud computing which has the advantages of high efficiency, high reliability, low cost, large-scale and deal with massive data of internet of things and create the basic conditions for the rapid development of the Internet of things.

We say that cloud computing is one of the basic conditions for the development of the Internet of Things,there is no cloud computing platform, the Internet of things is very difficult to get fast development,the reasons are as follows:The first: Cloud computing is the computer processing center of mass data in the Internet of things. There are three basic conditions for establishing internet of things.The first,Sensing equipment,there are some equipment to easily identify and collect information,such as: sensors, cameras, RFID, RFID tags, etc.Second,transmission network,these networks can transmit the information of interconnected object to processing center,and summarize, classification, analysis, processing,these networks will include our regular use of wired networks, wireless networks, satellite networks, etc;Third,the processing center of massive data,There must have a efficient data processing, large-scale, low-cost processing platform,It is to realize this process through cloud computing, in addition to real-time processing of data, Cloud Computing can also manage and store huge amounts of data in the Internet of Things.The second: Cloud computing can promote the effective combination of Internet of things,computing model and service delivery in the form of cloud computing can enhance the close interoperability between networking and the Internet, in order to build the so-called smart city, smart campus.

Cloud computing and the Internet of things have their own advantage,they combine to create a variety of different modes of application modes.For example:users can create smart Home Furnishing system to control and monitor all home appliances on the outside,and put a hot water bath temperature suitable when you aren't home; enterprises which have a distribution branch in multi citycan monitor each branch through the intelligent system of product quality.Of course, in addition to these, there are still a lot of cloud computing and networking application.

System framework of internet of things based on cloud computing. There are four levels of internet of things based on cloud computing framework,there are aware layer, network layer, the processing layer, the application layer[6],as shown in Figure.2. Main task is to collect information on the perception layer and convert format.The key technology of this level is the main sensor technology and wirelesssensor network technology,the network layer is based on the current mobile communication network, the Internet, etc,the key technology of this level mainly contains the network communication technology, as well as between the sensor network and communication network integration technology;processing layer to complete the data management, intelligent 
analysis,processing, and will process the data with the industry by combining,the key technology of this level includes the intelligent processing ability andcloud computing technology;the application layer is applied in different industries in the field of Internet of internet of things.In addition to these key technologies,applications of the Internet of things is to solve the technical standards between the sensor network interface, the sensor network and communication network access and other aspects of the problemAs well as technical secuity problems in collecting data,and to solve problem of the IP address of every object of internet of things[7].

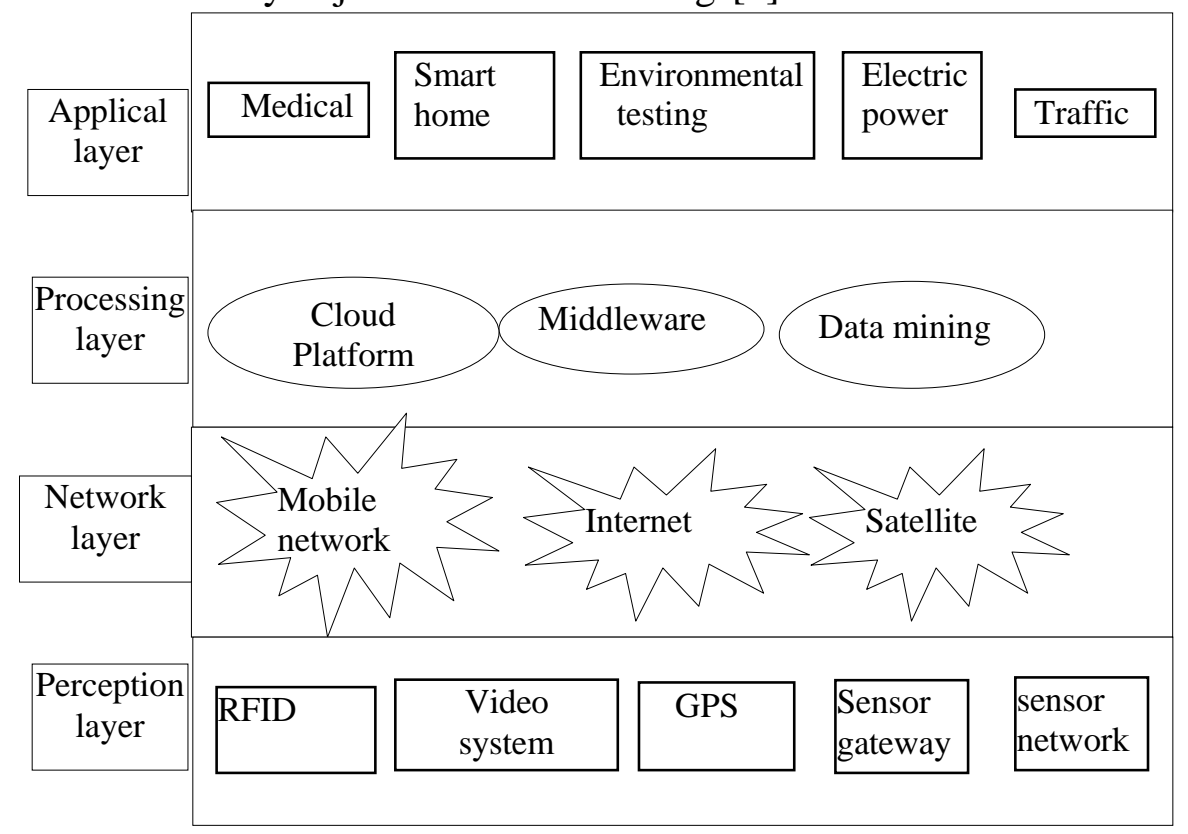

Fig.2 Networking framework based on Cloud Computing

The concept of internet of things from the start getted the concern of everyone, and quickly developed and the rapid application in various industries.Cloud computing With unique advantages achieves the rapid development of internet of things and provides technical support,it provide strong information processing capability for of objects of internet of things,application of cloud computing promotes large-scale application of the internet of Things.In the future development,we believe that , the application of internet of things will attract more users to participate in with the continuous development of both technologies mature.

\section{References}

[1] Jing Zhang."Current situation of international strategy and the study of Japanese physical network”,International News,September 2011.

[2] MichaelArmbrust et al.Albove the clouds:Aberkeley View of Cloud computing[R],Technical Report No.UCB/EECS-2009-28.

[3] Barrie Sosinsky.“Cloud Computing Bible”,Wiley press,December 1, 2010.

[4] Emmanuel Udoh.“Adoption of Grid and Cloud Computing Technology”,VDM Verlag Dr.

MullerAktiengesellschaft \& Co. KG press,July 9, 2010

[5] Sergey Andreev,SergeyBalandin,Yevgeni Koucheryavy.Internet of Things, Smart Spaces, and Next Generation Networking,Springer-Verlag Berlin andHeidelberg GmbH \& Co. K; 2012.

[6] onbo Zhou. The Internet of Things in the Cloud: A Middleware,CRC Press Inc press,October 29, 2012.

[7]Dieter Uckelmann, MarkHarrison, Florian.Michahelles Architecting the Internet of Things.Springer

Verlag Berlin and Heidelberg GmbH\&Co.K press,April 12,2011 